\title{
American Society of Hematology 2020 Podcast Collection: Graft-Versus-Host Disease
}

Jacopo Mariotti

Received: March 8, 2021 / Accepted: March 10, 2021 / Published online: March 30, 2021

(C) The Author(s) 2021

\section{PODCAST TRANSCRIPT}

David Gray (DG): Managing Editor of Advances in Therapy.

Jacopo Mariotti (JM): Bone Marrow Transplantation Unit, Humanitas Research Hospital, Milan.

DG: Hello, and welcome to the Adis Rapid+ podcast series. We're bringing you a selection of podcasts focused on the American Society of Hematology (ASH) 2020 Conference, discussing the highlights of the data released during the event. Today's podcast will be focusing on the graft-versus-host disease (GvHD) data as well as some other interesting findings which were presented at the ASH conference.

Speaking to us today is Dr. Jacopo Mariotti from the Bone Marrow Transplantation Unit of Humanitas Research Hospital in Milan. Jacopo, welcome to today's podcast, and thank you so much for speaking with us. First of all, would you like to give us a brief overview of the things that you'd like to cover today?

JM: Good morning first of all, and thank you for inviting me. And in this podcast, of course I

Supplementary Information The online version contains supplementary material available at https:// doi.org/10.1007/s12325-021-01706-w.

J. Mariotti $(\bowtie)$

Bone Marrow Transplantation Unit, Humanitas

Research Hospital, Milan, Italy

e-mail: jacopo.mariotti@cancercenter.humanitas.it will summarize the most relevant presentations held at the 62nd ASH annual meeting for the sessions concerning results in clinical allogeneic transplantation. So I will actually focus my presentation on three different topics: one on GvHD treatment and prophylaxis, the novelties; one about novelties about donor characteristics affecting the transplant's outcome; and my third one will be studies concerning comparison between allogeneic transplantation versus best available therapy for some hematologic diseases.

DG: Fantastic. That sounds brilliant. So to kick us off, what were some of the big announcements in terms of GvHD treatment?

JM: So one of the most expected results were the ones concerning the REACH3 trial [1]. So the REACH3 trial is a phase III study comparing ruxolitinib versus best available therapy for steroid-refractory and steroid-dependent chronic GvHD. An important point of this study is that the criteria used for... establishing the diagnosis of steroid-refractory and dependent chronic GvHD, as well as to measure disease response, are the ones that are actually recognized in the literature according to the EBMT (European Society for Blood and Marrow Transplantation) criteria and the NIH (National Institutes of Health) 2014 criteria.

So let's start from the study design. Patients were accrued with steroid-refractory and steroidchronic GvHD. They're actually randomized into 
one-to-one. One arm will receive ruxolitinib $10 \mathrm{mg}$ twice a day plus steroid plus calcineurin inhibitor. And the other arm will be treated with the best available treatment comprising steroid plus or minus calcineurin inhibitor; 165 patients will receive the ruxolitinib relative to 164 patients in the best available treatment arm.

A crossover was allowed on week 24 . So patients not responding to the best available treatment could cross over to the ruxolitinib arm. The primary endpoint was to measure the overall response rate at week 24 . And the key secondary endpoints were the failure-free survival and the measurement of improvement on clinical symptoms according to the modified Lee symptom scale on week 24 .

What was important results was that, first of all, the overall response rate in the ruxolitinib arm was much improved compared with the best available treatment arm. It was $76 \%$ relative to $60 \%$. In particular, $12 \%$ of the patients receiving ruxolitinib achieved a complete remission compared to $7 \%$ of patients receiving the best available treatment. This actually resulted in an improvement in the duration of response, because the median duration of response was not reached in the ruxolitinib arm relative to 6 months in the best available treatment arm. It was also orally reported by the researcher without-we did not see any data in the presentation-that the response rate for ruxolitinib was similar in all organs, including the lungs. Anyway, more data will be shown, hopefully, in the future.

And this improvement resulted also in better results for failure-free survival, because failurefree survival, median failure-free survival, was not reached in the ruxolitinib arm. It was about 6 months for the best available treatment arm. Failure-free survival... is defined in this study as a time from the randomization to relapse or progression for hematologic disease, non-relapse mortality, or the addition of a new systemic treatment for chronic GvHD. So this is actually a very meaningful endpoint, and ruxolitinib reached a very important result.

Looking at toxicity, the most frequent toxicity... was pretty manageable for ruxolitinib. The most frequent one was anemia and thrombocytopenia, with a grade 3 toxicity about $12 \%$ to $15 \%$ for the ruxolitinib relative to $7 \%$ to $10 \%$. The most important complicated side effect concerned fungal infections. The ruxolitinib arm fungal infection occurred in $11.5 \%$ of the patients relative to $6 \%$. And actually, grade 3 fungal infections were about $7 \%$ in the cohort receiving ruxolitinib relative to $2 \%$ for the best available treatment.

The researchers actually said that there was no difference in terms of overall survival and non-relapse mortality between the two arms, and that this was probably due to the increased chance of fungal infection in the ruxolitinib arm. Unfortunately, it's not well known which kind of fungal prophylaxis was held. But anyway, what we can conclude from this study is that ruxolitinib can actually improve the response in chronic GvHD, but fungal infection can be a concern. So we have to look... to improve fungal prophylaxis.

So there were other interesting studies reporting preliminary results for treatment for chronic GvHD. Because as we know, the clinical history of patients with chronic GvHD is very complex, since patients need to go through several different lines of treatment because at a certain point the disease becomes refractory or steroid-dependent. One preliminary... interesting study is the ROCKstar study, which is a phase II study employing belumosudil for retreatment of patients with chronic GvHD who actually received two to five prior lines of treatment [2].

So... what is belumosudil? This is actually a ROCK2 inhibitor. ROCK2 is actually a serine/ threonine kinase that plays a key role in immune disease. Especially ROCK2 inhibitions, so they're supposed to rebalance the immune system..., so it's possible to then regulate the pro-inflammatory Th17 cells while increasing the Treg cells.

Also, the ROCK signaling is important, because ROCK2 is involved not only to regulate the JAK-STAT signaling but also to regulate the multiple profibrotic processes, such as stress fiber formation and transcription of profibrotic genes.

So what about the study design? So inclusion criteria require a patient had a chronic GvHD, active chronic GvHD, with two to five prior 
lines of treatment. Two different doses were studied, were tested. So arm A will employ belumosudil $200 \mathrm{mg}$ every day. And the arm B will employ belumosudil $200 \mathrm{mg}$ twice a day; 66 patients were actually accrued in each arm.

The primary endpoint was to measure the overall response rate according to $2014 \mathrm{NIH}$ criteria. Results from this phase II study were very interesting, since the primary endpoint was actually met because overall response rate was above $70 \%$ for both dosages $-73 \%$ for one, $77 \%$ for the other one. Median time to response was pretty quick, 4 weeks. And a similar response rate was reported in all organs, including the fibrotic ones. Two-year overall survival was reported to be $81 \%$.

Another important point is that $64 \%$ of the patients were able to reduce their corticosteroid doses. And $21 \%$ were able to discontinue steroids. Moreover, $45 \%$ of the patients were able to reduce calcineurin inhibitor, and 25 were able to discontinue it. An improvement in clinical symptoms was actually measured by the Lee scale with an improvement in about $36 \%$ to $45 \%$ of the patients.

Another interesting study is a phase I/II study employing baricitinib for retreatment, again, of refractory chronic GvHD [3]. Baricitinib is a new JAK1/JAK2 inhibitor. In this phase I/II study, 20 patients were actually accrued, and 6 month overall response rate was actually interesting - $65 \%$ with a median time to response of 6 weeks; ... the researchers saw... some differences in response. So the highest organ-specific response was achieved in the lower gastrointestinal tract with $100 \%$ of response. But actually, only two patients had lower GI chronic GvHD. So it's difficult to say. This... needs to be confirmed with more patients. Another important response was actually found with a joint/fascia chronic GvHD with $85 \%$ response and with $50 \%$ of response for patients with mouth chronic GvHD.

Interestingly, no response was actually found for skin sclerotic GvHD, and very low for lung chronic GvHD, only 10\%. Steroid tapering actually occurred for $50 \%$ of the patients. And an improvement in clinical symptoms according to the Lee symptom scale was actually achieved in $50 \%$ of the patients. And also, overall survival was interesting. It was $100 \%$ at 1 year with a 1 -year failure-free survival of $74 \%$. This actually compares well with REACH3 study that was... about the same percentage.

Then another interesting study is a phase I study with employing axatilimab, again for retreatment of chronic GvHD for patients what have received at least two lines of previous treatment [4]. So axatilimab is actually an antibody against CSF-1 receptor.

CSF-1 is the colony-stimulating factor 1 , also named macrophage-stimulating factor 1 . So... CSF-1 and CSF-1 receptor actually regulate macrophage infiltration and cutaneous pathology. So this molecule is very important in the case of chronic GvHD, particularly in the sclerodermatous and pulmonary disease, because this is associated with the activity of macrophages that is CSF-1 receptor-dependent.

So what is expected in this study, what the researchers expected, that the treatment with an anti-CSF-1 receptor would deplete circulating non-classical monocytes, and also tissue macrophage infiltration, resulting in a reduction of GvHD-associated tissue pathology. Results were interesting. Fourteen patients were accrued with this phase I study but with an overall response rate of $57 \%$.

Response was similar among all analyzed groups; in particular, esophagus, the lower GI, and mouth sites showed a deep and sustained response, also in patients who were previously treated with ibrutinib and also ruxolitinib or a JAK inhibitor.

DG: Fantastic. Thanks, Jacopo. That was a brilliant summary. Now, you mentioned that you were also going to discuss prophylaxis. What was the big announcement around prophylaxis?

JM: About GvHD prophylaxis, we have at least three big retrospective registry studies concerning GvHD prophylaxis, all from the CIBMTR (Center for International Blood and Marrow Transplant Research). So one big study is... a retrospective study comparing the outcome of patients receiving haploidentical transplantation with PTCy (post-transplantation cyclophosphamide) relative to patients receiving allogeneic transplant from anotherfrom a different donor, such as a matched 
related donor transplant and matched unrelated donor, or a mismatched unrelated donor, or an umbilical cord blood donor [5]. This [was] for patients with acute lymphoblastic leukemia.

It's a large study comprising 4200 patients. Different types of GvHD prophylaxis were employed according to the different donor that was used. So PTCy was the main platform used for GvHD prophylaxis for haploidentical transplant, while calcineurin inhibitor plus methotrexate was the most frequent prophylaxis for matched related donor transplant. And calcineurin inhibitor plus methotrexate plus or minus ATG (anti-thymocyte globulin) was used for unrelated donor transplant. In case of a cord blood donor unit, calcineurin inhibitor was actually used with mycophenolate.

Results of this study are actually in line with the one actually reported in the literature, such as the one from Kanate or Fatobene $[6,7]$. So actually, no differences were actually found in terms of overall survival and leukemia-free survival between haploidentical transplant and HLA (human leukocyte antigen)-identical donor. But... an improvement in overall survival was found when comparing haploidentical donor relative to a mismatched unrelated donor or a cord blood unit. This improvement in overall survival was actually due to a reduced non-relapse mortality when the donor was a haploidentical donor compared either with a related donor, matched or mismatched unrelated donor, and also with a cord blood donor transplant. Non-relapse mortality was similar between haploidentical donor and matched related donor. And moreover, haploidentical donor had less frequently grade 2 to 4 acute GvHD compared with a related donor and cord blood donor.

And moreover, haploidentical donor had the lower incidence of chronic GvHD when compared with all the other platforms besides the cord blood donor. This report actually confirms and extends previous findings. But the problem was these studies that will actually compare in different donor but also different GvHD types of [and] different platform[s] of GvHD prophylaxis. So it would be interesting to know whether employing the same prophylaxis such as
PTCy in the setting of a matched unrelated donor transplant would bring different results.

And actually, a response... to this question comes from another study from CIBMTR, another big retrospective study, comparing the outcome of patients receiving haploidentical or matched related donor transplant and employing PTCy, so post-transplant cyclophosphamide-as GvHD prophylaxis [8]. So in the presentation, the researchers distinguished two different cohorts-one receiving myeloablative conditioning and one receiving a reduced-intensity conditioning.

About 1000 patients received myeloablative conditioning. And in this group, there were three... slightly different prophylaxis. So the haploidentical patients received PTCy plus calcineurin inhibitor plus mycophenolate, while for the matched unrelated donor transplant there were two different groups: one receiving the same prophylaxis PTCy, calcineurin inhibitor, and mycophenolate, and the other one was not receiving mycophenolate.

And as it is known from previous studies, such as the one by Mielcarek [9], avoiding mycophenolate... is actually bringing a higher incidence of GvHD. In fact, in the cohort of patients not receiving mycophenolate, these patients had an unacceptably high incidence of grade 2 to 4 acute GvHD that was actually 59\% for these patients relative to $43 \%$ of patients receiving mycophenolate, both in the haploidentical and in the matched unrelated donor transplant cohort.

This higher incidence of acute GvHD resulted in a higher 1-year non-relapse mortality for the matched unrelated donor transplant cohort not receiving mycophenolate, because it was $15 \%$ relative to haploidentical donor or matched unrelated donor transplant receiving mycophenolate as well. No differences were found in the other outcomes, such as chronic GvHD or overall survival.

Then there was another cohort of patients, about 1400 of patients receiving a reduced-intensity conditioning. Here the results were different because, first of all, we have only two cohorts. So one cohort was the haploidentical transplant receiving PTCy plus cyclosporin and 
plus a second calcineurin inhibitor plus mycophenolate. And the other cohort with actually the matched unrelated donor transplant was receiving exactly the same prophylaxis. So in this situation, no differences were found in terms of acute and chronic GvHD. It was about $24 \%$ to $29 \%$ for both donors.

But the most interesting result is that the non-relapse mortality was much higher for the haploidentical cohort relative to the matched unrelated donor cohort, because it was at 1 year $16 \%$ for haploidentical donor and $8 \%$ for matched unrelated donor transplant. And this brought a better 2-year overall survival and improved 2-year disease-free survival for patients receiving a matched unrelated donor transplant plus PTCy plus calcineurin inhibitor plus mycophenolate, because overall survival was $67 \%$ for a matched unrelated donor transplant relative to $54 \%$ for haploidentical. And disease-free survival was 54 relative to 41 .

Again, these results are actually in agreement with recent results from the EBMT registry that actually show similar results, similar, better, improved overall survival and lower non-relapse mortality when for matched related donor transplant and matched unrelated donor transplant relative to haploidentical transplant, when the same platform of GvHD prophylaxis is employed [10]. This improvement in overall survival and non-relapse mortality for the matched unrelated donor transplant is probably due to a later engraftment rate in the haploidentical arm, because patients receiving the haploidentical transplant had a later engraftment. And this actually brought a higher rate of fungal infection at 6 months. It was $10 \%$ relative to $1 \%$ for the patient receiving matched unrelated donor transplant.

What about employing the PTCy for unrelated donor transplant having one to four mismatched? So another study from the CIBMTR actually looked at PTCy GvHD prophylaxis for mismatched unrelated donor transplant with a seven out of eight, six out of eight, five out of eight, or four out of eight HLA mismatch [11]. There are a limited number of patients in this study, only 80 patients, with promising results, because 2-year overall survival was $79 \%$ for patients receiving reduced-intensity conditioning and $72 \%$ for receiving myeloablative conditioning. Non-relapse mortality was about the same, $20 \%$ for both cohorts.

Median time to engraftment was pretty good, because it was about 17 to 18 days, so a little delayed due to the post-transplant cyclophosphamide. Unfortunately, there was a high rate, high cumulative incidence of grade 2 to 4 acute GvHD. This was above $40 \%$ when the myeloablative conditioning was actually given. And of this percentage, $20 \%$ of the patients actually had 3 to 4 grade GvHD. And also, for reduced-intensity conditioning, 30\% of the patients had... grade 2 to 4 acute GvHD.

Also, high incidence of chronic GvHD disease was found in cases of myeloablative conditioning, about $40 \%$. It is important to notice that there was a low incidence of severe chronic GvHD. Types of infections were actually similar in these patients, similar to what we see in haploidentical transplants-so $10 \%$ at adenovirus infection, 5\% to $13 \%$ HHV-6. And there was a $30 \%$ of BK infection.

So death was also mostly due to relapse, followed by infections and multiorgan failure. So this study is interesting, because it shows that PTCy is an interesting and feasible platform for this type of transplant. But still, there is a high incidence of GvHD, especially when myeloablative conditioning is employed.

And other new strategies also were shown for GvHD prophylaxis. Especially, two interesting studies were reported. One results from the collaboration between Florida and the Minnesota centers, and GvHD prophylaxis consisted of a JAK2 inhibitor and an mTOR signaling blockade [12]. So this study actually comprised the usage of three different drugs. Tacrolimus was started on day minus 3 , sirolimus from day minus 1 , and pacritinib starting on day 0.

Pacritinib was tapered between day 70 and day 100. And this is a phase I study, where three-dose escalation steps were done with pacritinib starting from $100 \mathrm{mg}$ going to $200 \mathrm{mg}$ twice a day. Biological studies were reported in this trial, achieving one of their key points, their goals. What they found in the peripheral blood is that... the reduction of mTOR and the JAK2 activity was actually 
achieved, and also that STAT5 signaling was preserved. Blocking STAT3 signaling and maintaining STAT5 signaling resulted in a shift from Th17 to Treg phenotype in looking at, of course, the phenotype of $\mathrm{T}$ cells in the peripheral blood.

But the clinical results are very preliminary, because what we see is that 3 out of 12 patients had a grade 2 to 4 acute GvHD, and 3 out of 12 patients had mild chronic GvHD. Only one patient died because of steroid-refractory acute GvHD. Another interesting study concerning GvHD prophylaxis is the GRAVITAS-119, which is a phase I study employing itacitinib plus a calcineurin inhibitor for GvHD prophylaxis [13].

So inclusion criteria comprised patients receiving a matched related transplant or a matched unrelated donor transplant or a mismatched unrelated donor transplant. Prophylaxis consisted of tacrolimus plus methotrexate or cyclosporin plus mycophenolate plus or minus ATG-this is according to institutional decision-plus the addition of itacitinib. Itacitinib was actually employed as GvHD prophylaxis starting on day minus 3 up to day 180 . Reduced-intensity conditioning was actually used in this study; 65 patients were actually accrued.

The big problem of this study was the high rate of discontinuation, because $73 \%$ in the tacrolimus/methotrexate arm and $50 \%$ of the patients in the cyclosporin/mycophenolate arm discontinued their prophylaxis treatment. The main reason for discontinuation was actually disease relapse in this study. Anyway, the primary endpoint of this study was achieved, because no adverse effect on engraftment was seen, and engraftment was actually achieved in 64 out of 65 patients by day 28 post-transplant.

And when looking at the second endpoint, interesting results were seen, because acute GvHD rates were promising, because they ranged between 0 to $13 \%$. And similarly for looking at moderate to severe chronic GvHD, the cumulative incidence was pretty low, ranging between $12 \%$ to $36 \%$. Both of these acute and chronic GvHD incidence compare well with historical results. And moreover, the research didn't find any increase in infection or relapse rates for these patients.
DG: Thank you, Jacopo. You really are summarizing these data brilliantly. And I know that our listeners will be getting a lot out of this. We've talked a lot about prophylaxis, and we've talked about some of the drugs data. What other presentations did you think were really important, and what did you learn?

JM: I think at least two other presentations were really important. One was concerning strategies to improve the outcome of patients. So one was actually comparing allogeneic transplantation versus the best available therapy for patients with myelodysplastic syndrome aged 50 to 75 years old [14]. This is a study from the Blood and Marrow Transplant Clinical Trial Network.

And also, it's an important study because, for example in the USA, allogeneic transplantation is not covered... by health insurance for patients with myelodysplastic syndrome. So in this study, allogeneic transplantation was compared to the best available therapies such as hypomethylating agent or other therapies according to the physician choice.

An interesting result was about the most important clinical outcomes. So a statistically significant benefit in terms of overall survival was seen for patients in [the] allogeneic arm. So 3 -year overall survival was $48 \%$ for allorecipients relative to $27 \%$ for the non-donor arm, for an absolute improvement of $21 \%$ in overall survival. Consistently, the leukemia-free survival was improved for patients receiving allogeneic transplantation, because it was $36 \%$ relative to $22 \%$ for the non-transplant arm for an absolute improvement of $15 \%$, favoring the allogeneic transplant.

Both overall survival and leukemia-free survival benefit in the allogeneic transplant arm were independent by important other variables, such as age. So it was independent from patient age, because a similar advantage for allogeneic transplant was seen for patients aged between 50 and 65 years old or for patients older than 65 years old.

Moreover, these advantages for allogeneic transplants were maintained independently from the duration of treatment. Sorry, the duration between the time elapsed between the diagnosis of myelodysplastic syndrome and the 
treatment. So... if the time elapsed between the transplant and the diagnosis was lower or higher than 3 months, there was no difference in terms of this advantage of overall survival.

The advantage of... allogeneic transplant was evident for patients with high or very high revised IPSS (International Prognostic Scoring System), while it was not evident for a patient having a revised IPSS that was... very low, low, or intermediate. No reduction in quality of life was actually seen for... transplanted patients.

DG: Fantastic. Finally, final question. When we were talking before, you mentioned about choosing the best donor. What did you learn about choosing the best donor?

JM: Yes. Another interesting topic is the choice of best donor for allogeneic transplant because we can... actually test if choosing a best donor would result in a better outcome of allogeneic transplantation. There is an interesting study concerning the clonal donor hematopoiesis, which was actually held by the Dana-Farber and the Johns Hopkins Institutes [15]. So an important question in this study is whether donor clonal hematopoiesis could affect the outcome in terms of disease relapse and the transplant side effects such as GvHD and infections.

First of all, in this study, the first observation was that clonal hematopoiesis is very common in older donors. So first of all, the researchers looked at clonal hematopoiesis in older donors, meaning donors older than 40 years of age. And what they saw is that the most frequent genes with a mutation at a variant allele fraction (VAF) higher than $0.01 \ldots$ are DNMT3A, TET2, and ASXL1. By using a multivariable model, they saw that the clonal hematopoiesis with mutation at above VAF 0.01 was associated with an improved progression-free survival.

In particular, this advantage was due to a specific cohort, that is the cohort... with DNMT3A mutation at VAF above 0.01. So what we saw is that the clonal hematopoiesis with DNMT3A mutations is associated with an improved outcome in terms of overall survival, progression-free survival, and reduced relapse rate of hematologic disease. No effect was seen for other categories of donor clonal hematopoiesis, such as TET2. So other genes were not actually important for the outcome of patients.

And another important observation is that this effect of clonal hematopoiesis was... dependent [on] other variables such as prophylaxis, because... when... PTCy was used as GvHD prophylaxis, there was no advantage for patients receiving a transplant from a donor with a clonal hematopoiesis with a DNMT3A mutation. And also... the donor age [was important], because... for... donors older than 40 years of age, bringing a DNMT3A clonal hematopoiesis, in this situation the outcome was similar to younger donors. But it was a donor that was younger than 40 years of age. And it was much better compared to an older donor without a DNMT3A mutation in this clonal hematopoiesis.

And moreover, donor p53 mutations in clonal hematopoiesis were found to be associated with an increased risk of post-transplant donorderived cell leukemia.

DG: Well, Jacopo, that's all we've got time for. Thank you so much. That was a brilliant roundup of all of the GvHD highlights from ASH 2020. Thank you for joining me today. I know this will be really helpful for our listeners. So please do look out for other podcasts in the ASH 2020 coverage collection.

JM: Thank you very much.

You can listen to more podcasts by subscribing to Adis Rapid+ podcast with your preferred podcast provider, or by visiting the website. A full list of declarations, including funding and author disclosure statements, can also be found on the journal website.

\section{DIGITAL FEATURES}

This article is published with digital features, including a podcast video and audio file, to facilitate understanding of the article. To view digital features for this article go to https://doi. org/10.6084/m9.figshare.14179103. 


\section{ACKNOWLEDGEMENTS}

Funding. This podcast has been developed independently through an unrestricted educational grant by Novartis. The journal's Rapid Service and Open Access Fees were funded by an unrestricted educational grant by Novartis.

Authorship. The author meets the International Committee of Medical Journal Editors (ICMJE) criteria for authorship for this article, takes responsibility for the integrity of the work as a whole, and has given their approval for this version to be published.

Disclosures. Jacopo Mariotti has nothing to disclose.

Compliance with Ethics Guidelines. This article does not contain any studies with human participants or animals performed by any of the authors.

Peer Review. Please note, contrary to the journal's standard single-blind peer review process, as a podcast this article underwent review by a member of the journal's Editorial Board.

Open Access. This article is licensed under a Creative Commons Attribution-NonCommercial 4.0 International License, which permits any non-commercial use, sharing, adaptation, distribution and reproduction in any medium or format, as long as you give appropriate credit to the original author(s) and the source, provide a link to the Creative Commons licence, and indicate if changes were made. The images or other third party material in this article are included in the article's Creative Commons licence, unless indicated otherwise in a credit line to the material. If material is not included in the article's Creative Commons licence and your intended use is not permitted by statutory regulation or exceeds the permitted use, you will need to obtain permission directly from the copyright holder. To view a copy of this licence, visit http://creativecommons.org/licenses/by$\mathrm{nc} / 4.0 /$.

\section{REFERENCES}

1. Zeiser R, Polverelli N, Ram R, et al. Ruxolitinib (RUX) vs best available therapy (BAT) in patients with steroid-refractory/steroid-dependent chronic graft-vs-host disease (cGVHD): primary findings from the phase 3, randomized REACH3 study. In: ASH 2020 conference. 2020. https://ash.confex. com/ash/2020/webprogram/Paper137694.html. Accessed 17 Jan 2021.

2. Cutler C, Lee SJ, Arai S, et al. Belumosudil for chronic graft-versus-host disease (cGVHD) after 2 or more prior lines of therapy: the Rockstar study (KD025-213). In: ASH 2020 conference. 2020. https://ash.confex.com/ash/2020/webprogram/ Paper139445.html. Accessed 17 Jan 2021.

3. Holtzman NG, Im A, Ostojic A, et al. Efficacy and safety of baricitinib in refractory chronic graft-versus-host disease (cGVHD): preliminary analysis results of a phase 1/2 study. In: ASH 2020 conference. 2020. https://ash.confex.com/ash/2020/ webprogram/Paper140392.html. Accessed 17 Jan 2021.

4. Arora M, Jagasia M, Di Stasi A, et al. Phase 1 study of axatilimab (SNDX-6352), a CSF-1R humanized antibody, for chronic graft-versus-host disease after 2 or more lines of systemic treatment. In: ASH 2020 conference. 2020. https://ash.confex.com/ash/ 2020/webprogram/Paper141553.html. Accessed 17 Jan 2021.

5. Wieduwilt MJ, Metheny L III, Zhang M-J, et al. Comparison of haploidentical donor hematopoietic cell transplantation using post-transplant cyclophosphamide to matched-sibling, matchedunrelated, mismatched-unrelated, and umbilical cord blood donor transplantation in adults with acute lymphoblastic leukemia: a CIBMTR study. In: ASH 2020 conference. 2020. https://ash.confex. com/ash/2020/webprogram/Paper137186.html. Accessed 17 Jan 2021.

6. Kanate AS, Mussetti A, et al. Reduced-intensity transplantation for lymphomas using haploidentical related donors vs. HLA-matched unrelated donors. Blood. 2016;127:938-47.

7. Fatobene G, Rocha V, St Martin A, et al. Nonmyeloablative alternative donor transplantation for Hodgkin and non-Hodgkin lymphoma: from the LWP-EBMT, Eurocord, and CIBMTR. J Clin Oncol. 2020;38(14):1518-26.

8. Gooptu M, St. Martin A, Romee R, et al. Comparison of outcomes after haploidentical relative and HLA matched unrelated donor transplantation with post-transplant cyclophosphamide containing Gvhd prophylaxis regimens. In: ASH 2020 
conference. 2020. https://ash.confex.com/ash/ 2020/webprogram/Paper137221.html. Accessed 17 Jan 2021.

9. Mielcarek M, Furlong T, O'Donnell PV, et al. Posttransplantation cyclophosphamide for prevention of graft-versus-host disease after HLA-matched mobilized blood cell transplantation. Blood. 2016;127:1502-8.

10. Sanz J, Galimard JE, Labopin M, et al. Post-transplant cyclophosphamide after matched sibling, unrelated and haploidentical donor transplants in patients with acute myeloid leukemia: a comparative study of the ALWP EBMT. J Hematol Oncol. 2020;13(1):46.

11. Shaw BE, Jimenez-Jimenez A, Burns LJ, et al. Bridging the gap in access to transplant for underserved minority patients using mismatched unrelated donors and post-transplant cyclophosphamide: a national marrow donor program/be the match (NMDP/BTM) initiative. In: ASH 2020 conference. 2020. https://ash.confex.com/ ash/2020/webprogram/Paper137677.html. Accessed 17 Jan 2021.

12. Pidala J, Walton $\mathrm{K}$, Elmariah $\mathrm{H}$, et al. Biological and clinical impact of JAK2/mTOR blockade in gvhd prevention: preclinical and phase I trial results. In:
ASH 2020 conference. 2020. https://ash.confex. com/ash/2020/webprogram/Paper133874.html. Accessed 17 Jan 2021.

13. Choe H, Shah NV, Chevallier P, et al. A single-arm, open-label phase 1 study of itacitinib (ITA) with calcineurin inhibitor (CNI)-based interventions for prophylaxis of graft-versus-host disease (GVHD; GRAVITAS-119). In: ASH 2020 conference. 2020. https://ash.confex.com/ash/2020/webprogram/ Paper140747.html. Accessed 17 Jan 2021.

14. Nakamura R, Saber W, Martens MJ, et al. A multicenter biologic assignment trial comparing reduced intensity allogeneic hematopoietic cell transplantation to hypomethylating therapy or best supportive care in patients aged 50-75 with advanced myelodysplastic syndrome: blood and marrow transplant clinical trials network study. In: ASH 2020 conference. 2020. https://ash.confex.com/ ash/2020/webprogram/Paper136828.html. Accessed 17 Jan 2021.

15. Gibson CJ, Kim HT, Murdock HM, et al. DNMT3A clonal hematopoiesis in older donors is associated with improved survival in recipients after allogeneic hematopoietic cell transplant. In: ASH 2020 conference. 2020. https://ash.confex.com/ash/ 2020/webprogram/Paper142925.html. Accessed 17 Jan 2021. 